\title{
Prenyldiphosphate synthase, subunit 1 (PDSS1) and OH-benzoate polyprenyltransferase (COQ2) mutations in ubiquinone deficiency and oxidative phosphorylation disorders
}

\author{
Julie Mollet, ${ }^{1}$ Irina Giurgea, ${ }^{1}$ Dimitri Schlemmer, ${ }^{1}$ Gustav Dallner, ${ }^{2}$ Dominique Chretien, ${ }^{1}$
} Agnès Delahodde, ${ }^{3}$ Delphine Bacq, ${ }^{4}$ Pascale de Lonlay, ${ }^{1}$ Arnold Munnich, ${ }^{1}$ and Agnès Rötig ${ }^{1}$

${ }^{1}$ INSERM U781 and Department of Genetics, Hôpital Necker-Enfants Malades, Paris, France. ${ }^{2}$ Department of Molecular Medicine and Surgery, Karolinska Hospital, Karolinska Institutet, Stockholm, Sweden. ${ }^{3}$ Institut de Génétique et Microbiologie,

UMR 8621 CNRS, Université Paris-Sud, Orsay, France. ${ }^{4}$ Centre National de Génotypage, Evry, France.

\begin{abstract}
Coenzyme $\mathrm{Q}_{10}\left(\mathrm{CoQ}_{10}\right)$ plays a pivotal role in oxidative phosphorylation (OXPHOS), as it distributes electrons among the various dehydrogenases and the cytochrome segments of the respiratory chain. We have identified 2 novel inborn errors of $\mathrm{CoQ}_{10}$ biosynthesis in 2 distinct families. In both cases, enzymologic studies showed that quinone-dependent OXPHOS activities were in the range of the lowest control values, while OXPHOS enzyme activities were normal. $\mathrm{CoQ}_{10}$ deficiency was confirmed by restoration of normal OXPHOS activities after addition of quinone. A genome-wide search for homozygosity in family 1 identified a region of chromosome 10 encompassing the gene prenyldiphosphate synthase, subunit 1 (PDSS1), which encodes the human ortholog of the yeast $C O Q 1$ gene, a key enzyme of $\mathrm{CoQ}_{10}$ synthesis. Sequencing of PDSS1 identified a homozygous nucleotide substitution modifying a conserved amino acid of the protein (D308E). In the second family, direct sequencing of $\mathrm{OH}$-benzoate polyprenyltransferase (COQ2), the human ortholog of the yeast $\mathrm{COQ} 2$ gene, identified a single base pair frameshift deletion resulting in a premature stop codon (c.1198delT, $\mathrm{N} 401 \mathrm{fs} X 415)$. Transformation of yeast $\Delta \operatorname{coq} 1$ and $\Delta \operatorname{coq} 2$ strains by mutant yeast COQ1 and mutant human $\mathrm{COQ} 2$ genes, respectively, resulted in defective growth on respiratory medium, indicating that these mutations are indeed the cause of OXPHOS deficiency.
\end{abstract}

\section{Introduction}

Oxidative phosphorylation (OXPHOS) deficiency constitutes a clinically and genetically heterogeneous group of inherited diseases. One of these diseases, coenzyme $\mathrm{Q}_{10}$ (CoQ $\mathrm{C}_{10}$, ubiquinone) deficiency, is a recently identified entity of particular theoretical and practical importance. $\mathrm{CoQ}_{10}$ transfers reducing equivalents from various dehydrogenases to complex III (ubiquinone cytochrome $c$ reductase) and acts as a transmembrane hydrogen carrier. $\mathrm{CoQ}_{10}$ also plays a critical role in antioxidant defenses (1). Primary $\mathrm{CoQ}_{10}$ deficiency is a rare, but possibly treatable, autosomal recessive condition with 3 major clinical presentations: (a) an encephalomyopathic form, characterized by exercise intolerance, mitochondrial myopathy, myoglobinuria, epilepsy, and ataxia (2-5); (b) a generalized infantile variant with severe encephalopathy and renal disease (6-8); and (c) an ataxic form, dominated by ataxia, seizures, cerebral atrophy, and/or anomalies of the basal ganglia $(9,10)$. Recurrence of the disease and/or consanguinity of the parents in some reported families suggest

Nonstandard abbreviations used: $C O Q 2, O H$-benzoate polyprenyltransferase; $\mathrm{CoQ}_{10}$, coenzyme $\mathrm{Q}_{10}$; DQ, decylubiquinone; FPP, farnesylpyrophosphate; G3PDH, glycerol 3 phosphate dehydrogenase; GPP, geranylpyrophosphate; OXPHOS, oxidative phosphorylation; PDSS1, prenyldiphosphate synthase, subunit 1; PP, pyrophosphate.

Conflict of interest: The authors have declared that no conflict of interest exists. Citation for this article: J. Clin. Invest. 117:765-772 (2007). doi:10.1172/JCI29089. an autosomal recessive mode of inheritance. This has been demonstrated by recent reports of $\mathrm{OH}$-benzoate polyprenyltransferase (COQ2) and prenyldiphosphate synthase, subunit 2 (PDSS2) mutation in 2 independent families $(11,12)$.

Little is known about $\mathrm{CoQ}_{10}$ biosynthesis in humans, but several genes have been identified in the human genome by homology with other organisms, especially the Saccharomyces cerevisiae yeast (Figure 1). In the present study, in an inbred family with $\mathrm{CoQ}_{10}$ deficiency manifesting as a multisystem disease with early-onset deafness, encephaloneuropathy, obesity, livedo reticularis, and valvulopathy, homozygosity mapping allowed the disease to be attributed to a homozygous missense mutation in PDSS1, the enzyme that elongates the prenyl side chain of coenzyme Q. Moreover, direct sequencing of various genes involved in ubiquinone biosynthesis in an unrelated patient with fatal infantile multiorgan disease detected a homozygous single base pair frameshift deletion in COQ2. This study extends our understanding of this newly recognized and possibly treatable cause of OXPHOS deficiency in humans.

\section{Results}

Enzymologic studies and quinone quantification. Assessment of individual OXPHOS enzyme activities in cultured skin fibroblasts of patient 1 revealed normal activity of complex II, complex III, and complex IV (Table 1). However, quinone-dependent activities 


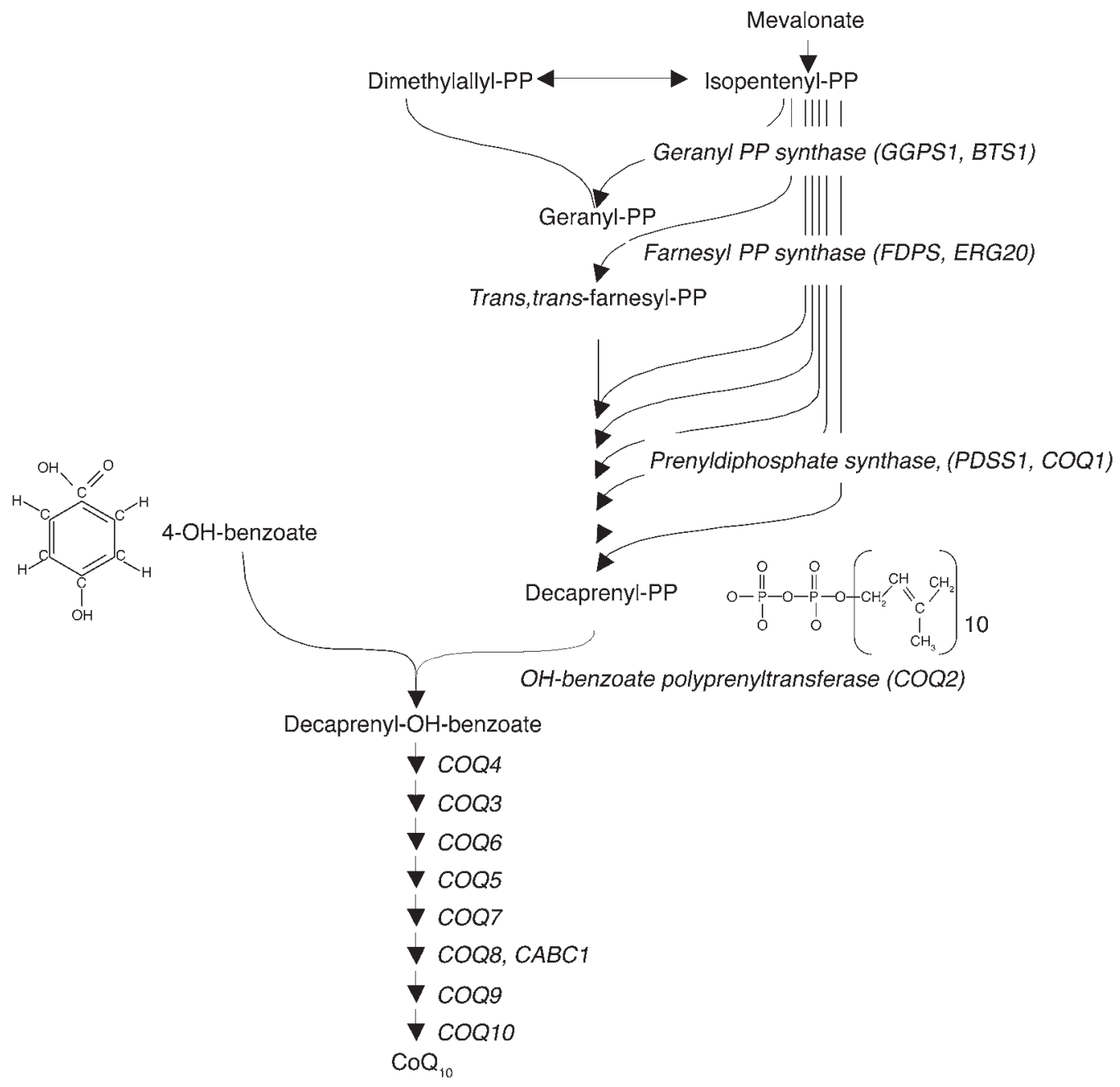

Figure 1

$\mathrm{CoQ}_{10}$ biosynthesis pathway. Enzyme and human gene symbols are shown in italics. The yeast gene symbol is indicated when different from the human gene.

$(\mathrm{CII}+\mathrm{CIII}$, glycerol 3 phosphate dehydrogenase $[\mathrm{G} 3 \mathrm{PDH}]+\mathrm{CIII})$ were in the range of the lowest control values, and activity ratios $(\mathrm{CIV} / \mathrm{CII}+\mathrm{CIII}, \mathrm{CII}+\mathrm{CIII} / \mathrm{G} 3 \mathrm{PDH}+\mathrm{CIII})$, which optimally detect unbalanced respiratory chain enzyme functions, were markedly altered compared with controls, suggesting quinone deficiency. Muscle mitochondria of patient 1 showed high absolute activity values for all complexes (Table 1) but abnormal activity ratios $(\mathrm{CI}+\mathrm{CIII} / \mathrm{CI}, \mathrm{CIII} / \mathrm{CI}+\mathrm{CIII})$, also indicating quinone deficiency. The hypothesis of ubiquinone deficiency was further supported by the dramatic effect of decylubiquinone (DQ, an exogenous ubiquinone analog) on succinate oxidation of fibroblasts from patient 1 , as addition of DQ during succinate oxidation measurement restored normal activity $(8$ and $16 \mathrm{nmol} / \mathrm{min} / \mathrm{mg}$ protein before and after DQ addition, respectively; normal values: $9.8-20.5 \mathrm{nmol} / \mathrm{min} / \mathrm{mg}$ protein) in the patient's permeabilized fibroblasts (Figure 2A). Consistently, addition of DQ during measurement of succinate-cytochrome $c$ reductase activity restored normal activity of cultured skin fibroblasts (19 and $44 \mathrm{nmol} / \mathrm{min} / \mathrm{mg}$ protein before and after DQ addition, respectively; normal values: $22-47 \mathrm{nmol} / \mathrm{min} / \mathrm{mg}$ protein; Figure $2 \mathrm{~B}$ ).
Finally, respiratory chain enzyme analysis in the liver of patient 3 revealed normal absolute enzyme activities but increased CIV/ $\mathrm{CII}+\mathrm{CIII}$ and $\mathrm{CIV} / \mathrm{CI}+\mathrm{CIII}$ activity ratios, also suggesting quinone deficiency (Table 1).

Direct evidence of quinone deficiency was finally provided by quantification of CoQ ${ }_{10}$ in the patients' fibroblasts, as the CoQ 10 content of the 3 patients' fibroblasts was markedly decreased compared with normal values (Table 2). Interestingly, a normal $\mathrm{CoQ}_{9}$ level was found in patients 1 and 2 . The markedly decreased $\mathrm{CoQ}_{10} / \mathrm{CoQ}_{9}$ ratio in patients 1 and 2 (0.3; controls: $\left.13 \pm 3\right)$ suggested a defective addition of the tenth prenyl to the polyprenyl chain. Fibroblasts from controls and patient 3 were grown in the presence of $\left[{ }^{3} \mathrm{H}\right]$ mevalonate to estimate the ability of these cells to synthesize $\mathrm{CoQ}_{10}$. Substantial incorporation of $\left[{ }^{3} \mathrm{H}\right]$ mevalonate into cholesterol, squalene, and dolichol was detected in controls and patient 3 , but no $\left[{ }^{3} \mathrm{H}\right] \mathrm{CoQ}_{10}$ was detected in cultured skin fibroblasts from patient 3 (Figure 2C). In these experiments, all lipid extracts were treated with acid phosphatase to dephosphorylate accumulated intermediates. In patient 3 , a peak with a retention time of 18.5 minutes was observed and was identi- 
Table 1

Respiratory chain enzyme activities in muscle mitochondria and cultured skin fibroblasts

$\begin{array}{cc}\text { Enzyme } & \text { Patient } 1 \\ \text { Cultured skin } \\ \text { fibroblasts }\end{array}$

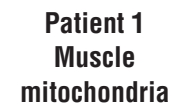

mitochondria

Activities (nmol/min/mg protein)

\begin{tabular}{|c|c|c|c|}
\hline $\mathrm{Cl}$ & - & $279(65.2 \pm 16.6)$ & $33(28.6 \pm 6.3)$ \\
\hline Cll & $14(20.1 \pm 3.5)$ & - & $202(158.5 \pm 28.1)$ \\
\hline CIII & $123(169.6 \pm 37.5)$ & $3,838(1,258.8 \pm 327.5)$ & $327(250.8 \pm 40.6)$ \\
\hline CIV & $93(107.1 \pm 25.6)$ & $1,827(730.3 \pm 233.4)$ & $364(270.6 \pm 48.3)$ \\
\hline CV & - & - & $114(96.2 \pm 32.1)$ \\
\hline $\mathrm{Cl}+\mathrm{CIII}$ & - & $414(199 \pm 56.4)$ & $25(67.5 \pm 16.5)$ \\
\hline $\mathrm{Cll}+\mathrm{CIII}$ & $19(33.4 \pm 6.7)$ & $596(228.8 \pm 61.8)$ & $25(69.5 \pm 15.4)$ \\
\hline G3PDH & $13(14.4 \pm 2.5)$ & - & - \\
\hline G3PDH+CIII & $5(18.2 \pm 3.5)$ & $43(38.6 \pm 11.6)$ & - \\
\hline \multirow[t]{2}{*}{ CS } & $71(77 \pm 20.7)$ & $139(324.1 \pm 92.9)$ & $74(102.5 \pm 15.9)$ \\
\hline & & Activity ratios & \\
\hline $\mathrm{CIV} / \mathrm{Cl}$ & - & $4.4(3.5 \pm 0.9)$ & $11(8.8 \pm 1.8)$ \\
\hline $\mathrm{CIV} / \mathrm{CII}$ & $6.6(5 \pm 0.5)$ & - & $1.8(1.5 \pm 0.4)$ \\
\hline CIV/CIII & $0.8(0.6 \pm 0.01)$ & $0.5(0.5 \pm 0.04)$ & - \\
\hline CIV/CV & - & $2.3(2.5 \pm 0.3)$ & $3.2(2.0 \pm 0.4)$ \\
\hline $\mathrm{CIV} / \mathrm{CII}+\mathrm{CIII}$ & $4.8(3 \pm 0.2)$ & $3.1(3.2 \pm 0.3)$ & $14.6(2.8 \pm 0.6)$ \\
\hline $\mathrm{CIV} / \mathrm{Cl}+\mathrm{CIII}$ & - & $4.4(3.4 \pm 0.9)$ & $14.6(3.6 \pm 0.9)$ \\
\hline $\mathrm{Cl}+\mathrm{ClII} / \mathrm{Cl}$ & - & $1.5(3.3 \pm 0.7)$ & - \\
\hline $\mathrm{CII}+\mathrm{CIII} / \mathrm{G} 3 \mathrm{PDH}+\mathrm{CIII}$ & | $3.7(1.4 \pm 0.1)$ & $13.6(5.5 \pm 1.3)$ & - \\
\hline $\mathrm{CIII} / \mathrm{Cl}+\mathrm{CIII}$ & - & $\mathbf{9 . 3}(6.5 \pm 1.6)$ & $13.08(3.01 \pm 0.52)$ \\
\hline $\mathrm{Cl} / \mathrm{CS}$ & - & - & $0.44(0.38 \pm 0.07)$ \\
\hline $\mathrm{CIV} / \mathrm{CS}$ & $1.3(1.37 \pm 0.21)$ & - & $4.9(3.1 \pm 0.39)$ \\
\hline $\mathrm{Cll}+\mathrm{Cll} / \mathrm{CS}$ & $0.26(0.47 \pm 0.07)$ & - & $\mathbf{0 . 3 3}(1.07 \pm 0.16)$ \\
\hline
\end{tabular}

Abnormal values are shown in bold; control values are shown in parentheses; and ratio values are presented as mean $\pm 1 \mathrm{SD}$. $\mathrm{Cl}$, complex I; CS, citrate synthase.

\section{Patient 3 \\ Liver homogenate}

33

$202(158.5 \pm 28.1)$

$327(250.8 \pm 40.6)$

$114(96.2+32.1)$

$25(67.5 \pm 16.5)$

$\pm 15.4)$

$\pm 15.9)$

$11(8.8 \pm 1.8)$

$5 \pm 0.4)$

$3.2(2.0 \pm 0.4)$

$14.6(2.8 \pm 0.6)$

$-$

$.08(3.01 \pm 0.52)$

$44(0.38 \pm 0.07)$

$0.33(1.07 \pm 0.16)$

a glutamic acid (D308E). This aspartic acid is also conserved in eukaryote and prokaryote farnesylpyrophosphate (FPP) synthases and geranylpyrophosphate (GPP) synthases (13-15). Moreover, this aspartic acid lies in the signature pattern of polyprenyl synthase ([LIVMFY]-G-x(2)-[FYL]-Q-[LIVM]-x-D-D[LIVMFY]-X-[DNG]), an aspartic acid-rich region of the protein that could be involved in the catalytic mechanism and/or binding of the substrates (Figure 4E). Both parents were heterozygous for the $\mathrm{T} \rightarrow \mathrm{G}$ transversion (Figure 4B), and unaffected children were either heterozygous or homozygous for the wild-type allele. The $\mathrm{T} \rightarrow \mathrm{G}$ transversion created an MnlI restriction site. Exon 10 was amplified, and the PCR product was then digested by the restriction enzyme MnlI. This generated 2 fragments in controls (393 and $180 \mathrm{bp}$ ) and 3 fragments in patients $(352,180$, and $41 \mathrm{bp}$; Figure 4D). This mutation was absent from 100 controls of the same ethnic origin.

Finally, direct sequencing of the genes known to be involved in ubiquinone biosynthesis was systematically performed in patient 3 and identified a homozygous base pair deletion in exon 7 of the OH-benzoate polyprenyltransferase gene (COQ2, c.1198delT, N401fsX415; Figure 5A) resulting in a premature stop codon. Both parents were heterozygous for this single nucleotide deletion (Figure 5B), and the deletion was absent in 100 controls of the same ethnic origin. RT-PCR

fied as decaprenol (Figure 2C). Decaprenyl-pyrophosphate (PP) accumulation reflected a deficiency in COQ2, which conjugates decaprenyl-PP with the benzoquinone ring.

Molecular studies. A genome-wide search for homozygosity was performed in the family of patients 1 and 2 using 400 polymorphic markers. This study identified 6 chromosomal regions of homozygosity on chromosomes 1 (D1S2762-D1S431, $3.9 \mathrm{cM}), 7$ (D7S2426-D7S2462, 9.4 cM), 10 (D10S1714-D10S593, $9.6 \mathrm{cM}$ ), 12 (D12S1608-D12S1685, $5 \mathrm{cM}$, and D12S1725-D12S77, 11.3 $\mathrm{cM}$ ), and 13 (D13S280-D13S286, 8.4 cM). The 10p12.2-p12.1 region (Figure 3 ) encompasses a candidate gene, PDSS1, encoding the prenyldiphosphate synthase, subunit 1 , which elongates the prenyl side chain of coenzyme $\mathrm{Q}$ in the quinone biosynthesis pathway $(1,13)$ (Figure 1$)$. A mutation search was therefore undertaken in the family by direct sequencing of the 12 exons of the PDSS1 gene. A homozygous $\mathrm{T} \rightarrow \mathrm{G}$ transversion at nucleotide 977 was found in exon 10 (Figure 4A). This transversion resulted in the change of a highly conserved aspartic acid into

\section{Figure 2}

Effect of an exogenous quinone analog (DQ, $80 \mu \mathrm{M})$ on succinate oxidation (A) and succinate cytochrome $c$ reductase activity of cultured skin fibroblasts from patient 1 (B). Numbers along the traces represent $\mathrm{nmol} / \mathrm{min} / \mathrm{mg}$ protein. Numbers in brackets are normal values. A, absorbance. (C) Elution profiles of lipid extracts from control and patient 3 skin fibroblasts incubated with $\left[{ }^{3} \mathrm{H}\right]$ mevalonate and treated with acid phosphatase. amplification on fibroblast RNAs showed that the abnormal transcript was still present in patient 3 (Figure 5C). This result suggests that a shortened mutant protein (364 instead of 371 amino acids with $14 \mathrm{C}$-proximal residues differing from the

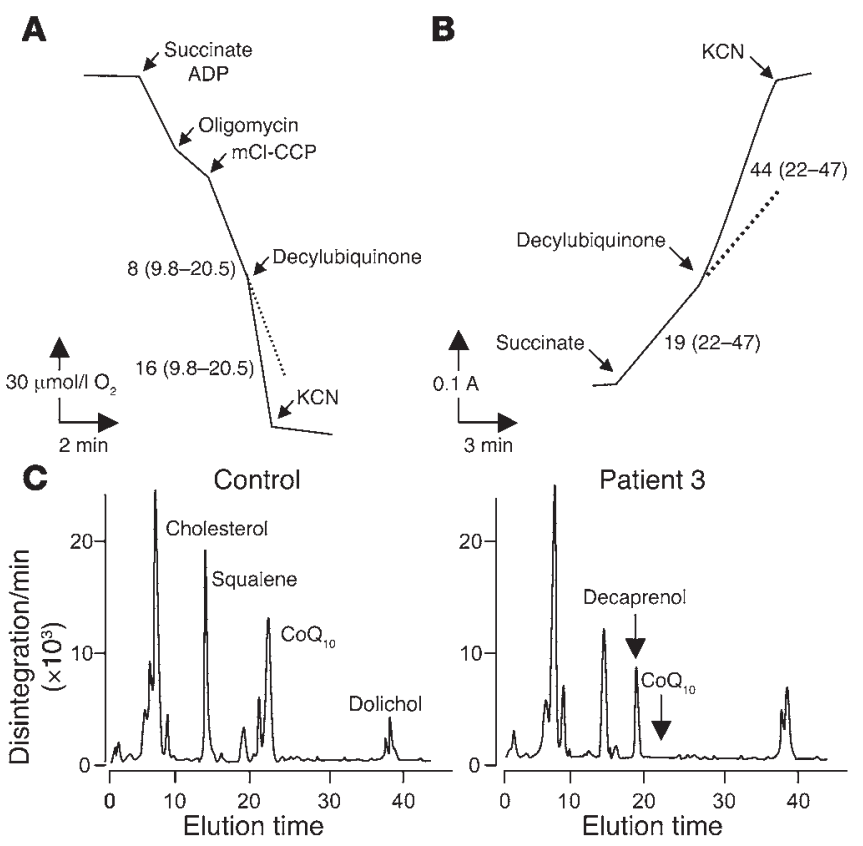


Table 2

Quinone content in cultured skin fibroblasts

\begin{tabular}{|c|c|c|c|c|}
\hline & Patient 1 & Patient 2 & Patient 3 & $\begin{array}{l}\text { Control } \\
(n=13)\end{array}$ \\
\hline $\mathrm{CoQ}_{10}(\mathrm{nmol} / \mathrm{g}$ protein) & 4 & 4 & 29 & $120 \pm 32$ \\
\hline $\mathrm{CoQ}_{9}$ (nmol/g protein) & 12 & 11 & 3 & $10 \pm 2$ \\
\hline $\mathrm{CoQ}_{10} / \mathrm{CoQ}_{9}$ & 0.33 & 0.36 & 9.66 & $13 \pm 3$ \\
\hline
\end{tabular}

Abnormal values are shown in bold.

normal protein) is synthesized in this patient. Unfortunately, no anti-human Coq2 antibody was available to test this hypothesis. Moreover, the global charge of the C-proximal part of the mutant protein was also modified (Figure 5D).

Functional complementation. To confirm the deleterious nature of the D308E PDSS1 genotype, the human wild-type and mutant genes were tested for their ability to complement the OXPHOS defect in the Saccharomyces cerevisiae strain deleted for the COQ1 gene. Wild-type and mutant PDSS1 cDNAs as well as the yeast COQ1 gene were cloned in pYES2.1. The 3 different plasmids were used to transform a yeast $\Delta \operatorname{coq} 1$-null strain. Growth of the mutant on glycerol-rich medium was restored by the yeast COQ1 gene but not by wild-type or mutant human PDSS1 cDNAs, demonstrating that human prenyldiphosphate synthase does not complement the yeast $\Delta$ coq1-null mutation (Figure $6 \mathrm{~A}$ ). The mutation was then introduced into the yeast $C O Q 1$ gene by site-directed mutagenesis. The yeast D365E mutant protein (corresponding to the human D308E protein) failed to complement the yeast mutant, whereas the normal protein did, demonstrating that the mutation affects protein function (Figure 6A).

To assess the deleterious nature of the $\mathrm{OH}$-benzoate polyprenyltransferase gene mutation, the yeast $\Delta$ coq2-null strain was transformed with either normal or mutant human cDNAs cloned in pYES2.1. The human wild-type cDNA restored growth on glycerol-rich medium, whereas the mutant protein did not, showing that the mutation is indeed the cause of the deficiency (Figure 6B).

\section{Discussion}

This study reports on the identification of deleterious mutations in 2 different genes of the ubiquinone biosynthesis pathway (PDSS1 and $C O Q 2$ ), which caused severe ubiquinone deficiency in 2 unrelated families. In the first consanguineous family, a genome scan analysis identified a homozygous mutation in the PDSS1 gene encoding prenyldiphosphate synthase, one of the key enzymes of the ubiquinone biosynthesis pathway. This enzyme catalyzes elongation of GPP or FPP with several isopentenylpyrophosphate (IPP) groups, in trans configuration, to form the isoprenoid chain $(6,13)$. The mutation reported here changed a highly conserved aspartic acid into a glutamic acid (D308E). Human wild-type PDSS1 cDNA failed to complement the OXPHOS defect of the $\Delta$ coq1-null yeast strain, while the PDSS1 and Coq1p proteins share $35 \%$ homology. However, growth of the $\Delta \operatorname{coq} 1$ yeast strain on a nonfermentable carbon source was restored by the wild-type but not the mutant yeast gene (D365E, corresponding to human D308E). We therefore conclude that the D308E mutation in our patient clearly induced prenyldiphosphate synthase deficiency and a profound quinone biosynthesis defect. In Saccharomyces cerevisiae, Coq1p (hexaprenylsynthase) elongates GPP or FPP (diprenyl biosynthesis intermediaries) to form hexaprenylpyrophosphate. The exact function of the PDSS1 protein in humans is not well known, but it can be hypothesized, by homology with yeast, that the PDSS1 enzyme also elongates geranyl pyrophosphate to form decaprenyl pyrophosphate. However, the normal $\mathrm{CoQ}_{9}$ but very low CoQ 10 levels detected in patients 1 and 2 suggest that the D308E PDSS1 mutation only impairs addition of the tenth prenyl to the polyprenyl chain. It is worth noting that quinone-dependent OXPHOS activities were only slightly decreased in patients 1 and 2 , despite profound $\mathrm{CoQ}_{10}$ depletion in fibroblasts. It can be hypothesized that the mutant PDSS1 retains a low but significant residual activity, allowing the synthesis of trace amounts of $\mathrm{CoQ}_{10}$ and/or normal amounts of $\mathrm{CoQ}_{9}$ sufficient to maintain a residual respiratory chain function compatible with life.

The mutated amino acid residue (D308) is situated in the second aspartic acid-rich region of the protein, which is common to all prenylsynthases reported to date. This family of proteins also includes FPP synthase and GPP synthase, 2 other enzymes of the ubiquinone synthesis pathway (13-15). Prenylsynthases are also involved in the biosynthesis of terpenoids in plants (16) and carotenoids in fungi (17). These enzymes are also involved in protein farnesylation (18). The aspartic acid-rich prenylsynthase signatures are supposedly involved in binding of the substrate, isopentenyl pyrophosphate, and elongating dimethylallyl pyrophosphate, by forming magnesium salt bridges between the substrate and the catalytic site (13). Site-directed mutagenesis of the second aspartate-rich motif has been performed in FPP synthase from Saccharomyces cerevisiae (19) and Bacillus stearothermophilus (20). These data show that the FPP synthase aspartate residue

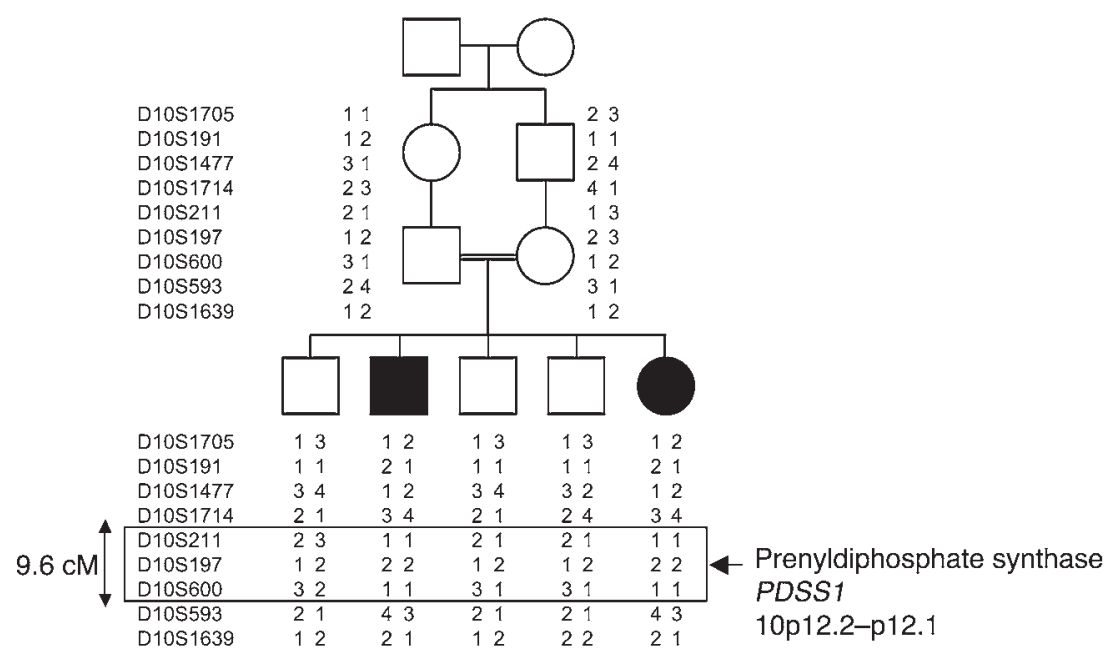

Figure 3

Pedigree and haplotype analysis of the family of patients 1 and 2. Haplotypes are given (top to bottom) for loci D10S1705, D10S191, D10S1477, D10S1714, D10S211, D10S197, D10S600, D10S593, and D10S1639. The haplotypes in the upper part are from the parents. 


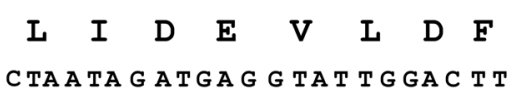
C TA A TA G A T A G G

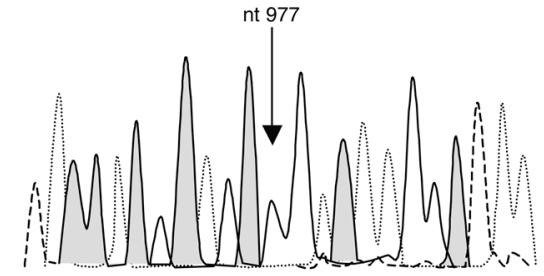

$\begin{array}{lllllllll}L & I & D & D / E & V & L & D & F\end{array}$ CTAATAGATGA TT GATTGGACTT

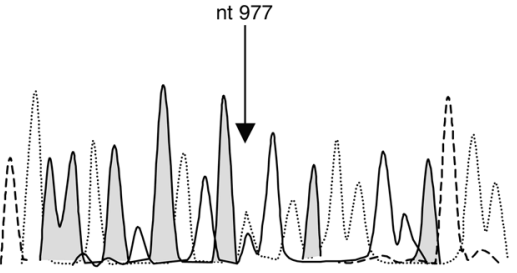

C $\begin{array}{llllllll}I & I & D & D & V & L & D & F\end{array}$ CTAATAGATGAT TATTGGACTT

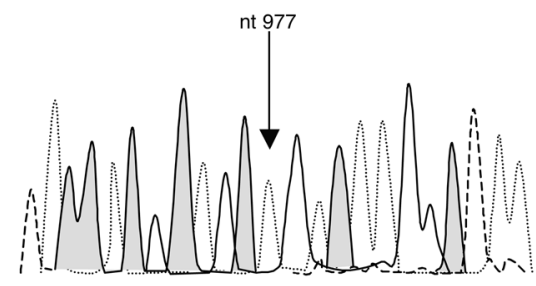

D

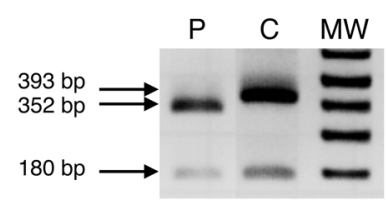

D308E

Prenyldiphosphate synthase
$E . \quad$ COIi

H. influenzae KALQDYGRYLGTAFQLVDDVLDYSANTQAL

B. subtilis KGLQDYGRYLGTAFQLIDDLLDYNADGEQI

. crassa VDAYYYGKITGIAFOLVDDMT DYTRSEKE

crassa VDAAYYG NLGLAFQLVDDMYDYTRSEKE

pombe TAAGEYGROIGTAEQLMDDVLDYTSKDDT

. cerevisiae DECYDFGRN LGICEQLVDDMLDETVSGKDL

A. thaliana VLAFEYGRN LGLAFOLIDDILDETGTSASL

D. melanogasterEVAFQYGRN IGLAFQLVDDMLDEVSSTEQM

M. musculus EIAYQYGKNVGIAEQLIDDVLDETSCSDQM

$H$. Sapiens EIAYQYGKNVGIAFQLIDDVLDFTSCSDQM

E. Coli

- crassa KQAEDILIALGEYEQVQDDYLDNFGLPEHI

. pombe KCAQDILIILGKYFQVQDDYLD CYGDPTVT

S. cerevisiae KQARDVLIALGEYFQIQDDYLDCFGTPEQI

A. thaliana IDVKNVLVDMGIYFQVQDDYLDCFADPETL

D. melanogasterRQSKTILLEMGNFFQVQDDFLDCFGNPEVT

M. musculus ANALKILMAMGEFFOVODDYLD LFGDPSUT

M. MUsculus ANALKILM

GPP sapiens

E. COIi

H. influenzae QSLTQYAEA IGLAFQVQDDILDIEGDSAEI

3. subtilis ETLRTFSSH IGIGEQIRDDILDLEGSEEKI

N. crassa VDCVPLVNIIGLIFQIADDYHN LWNREYTA

S. cerevisiae HSLVPFINILGIIYQIRDDYLN LKDFQMSS

A. thaliana ERLRKFARdIGLLFQVVDDILDVTKSSKEI

DYCNLSLKEVSV

M. musculus EDLKPLLDTLGLFFQIRDDYANLHSKEYSE

H. sapiens EDLKPLLNLLGLFEQIRDDYANLHSKEYSE

[LIVMFY]-G-X (2)-[FYL]-Q-[LIVM]-X-D-D-[LIVMFY]-X-[DNG]

Polyprenyl synthase signature

Figure 4

Molecular analysis of the PDSS1 gene. Sequence analysis of PDSS1 gene in patient 1 (A), parents (B), and controls (C). (D) Mnll restriction analysis of the $T \rightarrow G$ mutation at nt 977 . Mnll restriction generated 2 fragments of 393 and $180 \mathrm{bp}$ in controls and 3 fragments of 352 , 180, and $41 \mathrm{bp}$ in the patient. P, patient; C, controls; MW, molecular weight marker (DNA molecular weight marker VIII; Roche Applied Science). (E) Sequence alignment of the prenyldiphosphate synthase, FPP synthase, and GPP synthase from human and nonhuman sources. The box shows the polyprenyl synthase signature.

corresponding to the human PDSS1 residue D308 is essential for catalytic activity. Moreover, the mutagenesis of aspartate to glutamate at position 244 of the rat FPP synthase (D244E), equivalent to the D308E in PDSS1, resulted in a 7-fold reduction in the $V_{\max }$ value of the enzyme (21). The D308E mutation identified in our patients with ubiquinone deficiency therefore confirms the functional importance of this particular residue for the prenylsynthase activity of the protein.

The OH-benzoate transpolyprenyltransferase (COQ2) gene encodes the enzyme involved in the second step of ubiquinone biosynthesis $(13,22)$. In a second family with ubiquinone deficiency, a single base pair deletion was identified in the COQ2 gene, resulting in a premature stop codon and modifying the last 21 amino acids of the protein. The normal but not the mutant protein rescued the growth defect of the $\Delta c_{c o q} 2$ yeast strain on a nonfermentable carbon source, therefore demonstrating the deleterious consequences of the mutation. Finally, the abnormal accumulation of decaprenyl-PP, the substrate of $\mathrm{OH}$-benzoate polyprenyltransferase in cultured skin fibroblasts of the patient, also supports the relevance of this mutation in ubiquinone deficiency. The mutated domain of the protein is not well conserved across species, but the mutation modifies the global charge at the C-terminal end of the protein, possibly altering its interactions with other proteins. Several enzymes involved in ubiquinone biosynthesis in yeast have been shown to form a multisubunit complex, as Coq1p, Coq4p, Coq5p, and Coq6p have been shown to form a high-molecular-weight complex $(23,24)$. It is not yet known whether Coq2p is also involved in this complex in yeast and whether a similar complex exists in humans. Never- theless, the deleterious effect of this human mutant protein in yeast suggests that various interactions between the proteins of the 2 species are still retained despite the absence of sequence homology between human and yeast $\operatorname{Coq} 2 \mathrm{p}$ at the C-terminal ends of the proteins.

Primary ubiquinone deficiency is a rare cause of mitochondrial disorders, and the genetic bases of these disorders have rarely been identified, as COQ2 or PDSS2 mutations have only been reported twice in patients with encephalomyopathy and kidney involvement $(11,12)$. In this article, we report what we believe to be the first molecular/functional characterization of PDSS1 and COQ2 mutations and demonstrate that both mutations are disease causing.

It is noteworthy that optic atrophy, deafness, obesity, pancytopenia, and cardiac disease have not been previously reported in primary ubiquinone deficiency. While optic atrophy, deafness, and pancytopenia are occasionally reported in respiratory chain deficiency, it remains unclear whether obesity and valvulopathy are directly related to ubiquinone deficiency. Similarly, early and severe forms of ubiquinone deficiency, fatal in the early neonatal period, have seldom been reported (7). Why profound ubiquinone deficiency in cultured fibroblasts was associated with either neonatal death or long survival also remains unexplained (patients 1 and 2 were 22 and 14 years old, respectively). The clinical heterogeneity of ubiquinone deficiency is suggestive of a genetic heterogeneity that could be related to the large number of enzymes and corresponding genes involved in ubiquinone biosynthesis. It is hoped that identification of disease-causing genes in other families will help to elucidate the clinical variability of these rare conditions. 
A
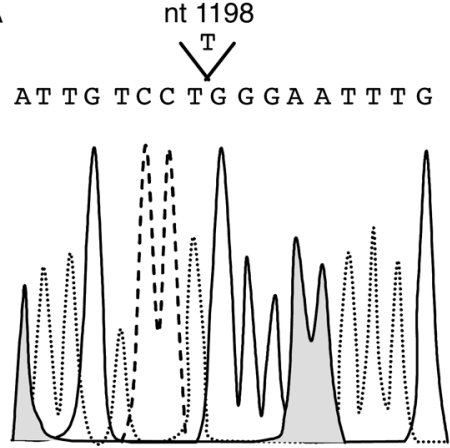

D

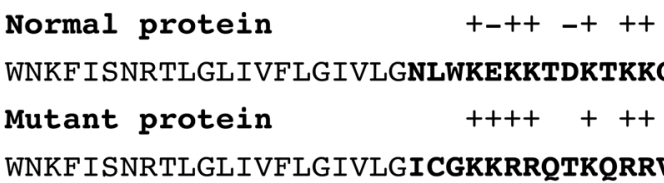

B
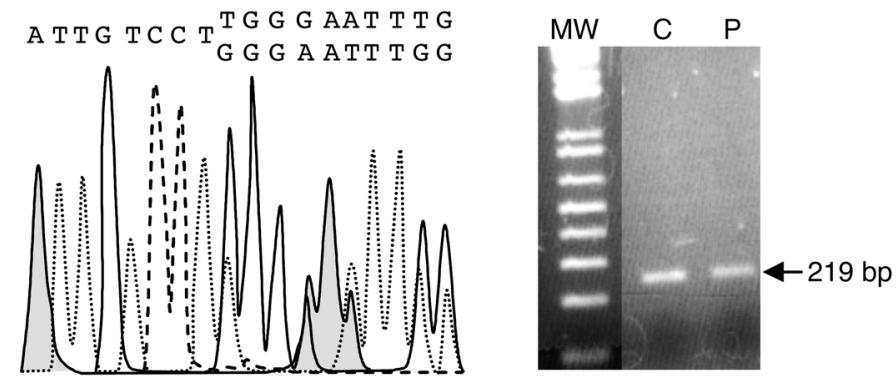

\section{Figure 5}

Molecular analysis of the COQ2 gene. Sequence analysis of the COQ2 gene in patient 3 (A) and her parents (B). (C) RT-PCR analysis of COQ2 transcript. (D) Normal and mutant coq2 proteins.

\section{Methods}

Patients. We obtained informed consent from parents and all probands and siblings before collecting blood for DNA extraction or performing tissue biopsies. Patient 1 , a boy, was born to first-cousin healthy Moroccan parents. He had 3 unaffected brothers and 1 affected sister. He was normal at birth (weight, $2.7 \mathrm{~kg}$; height, $47 \mathrm{~cm}$; head circumference, $35 \mathrm{~cm}$ ). Deafness was detected at 1 year of age, and he developed bulimia, obesity, livedo reticularis, and optic atrophy at 3 years. Valvulopathy characterized by mild aortic and mitral regurgitation with moderate pulmonary artery hypertension was detected at the age of 15 . At the time of publication, he was 22 years old, with mild mental retardation, and, unlike his healthy siblings, he presented overweight (weight, $73 \mathrm{~kg}+1.5 \mathrm{SD}$; height, $1.58 \mathrm{~m}-2 \mathrm{SD}$ ) and macrocephaly (head circumference, $57 \mathrm{~cm}+1.5 \mathrm{SD}$ ). He also has a peripheral neuropathy with absent deep tendon reflexes. Mildly elevated plasma lactate (2.1 mmol/1; N, 1.0-1.55) and lactate/ pyruvate molar ratios (16; N, 6-14) were observed, and muscle biopsy showed mitochondrial aggregates.

Patient 2, his sister, was normal at birth (weight, $2.9 \mathrm{~kg}$; height, $46 \mathrm{~cm}$; head circumference, $34 \mathrm{~cm}$ ). She also developed deafness in her second year and valvulopathy at the age of 9 years. At the time of publication, she was 14 years old and presented obesity (weight, $75 \mathrm{~kg}+4 \mathrm{SD}$; height, $133 \mathrm{~cm}$ ), macrocephaly (head circumference, $57 \mathrm{~cm}+3 \mathrm{SD}$ ), optic atrophy, periph-
$\mathbf{A}$
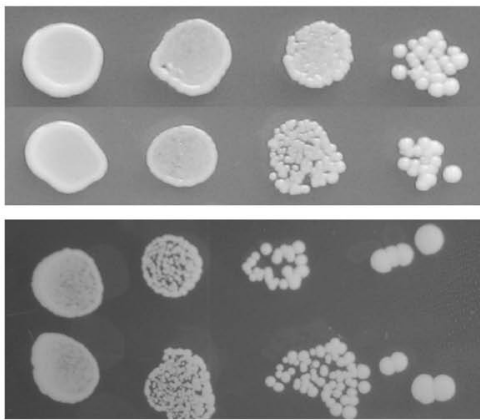

B

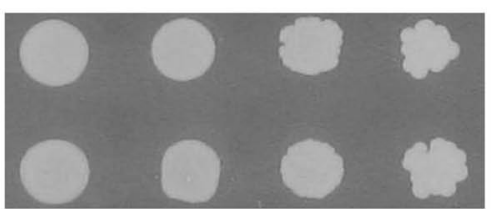

WO-HLM

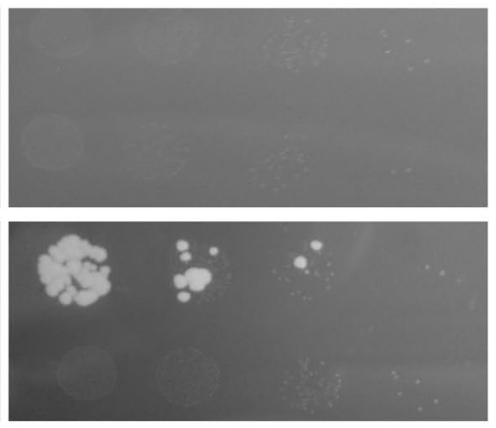

$\triangle \operatorname{coq} 1+$ pYES2.1-WT PDSS1

$\Delta c 0 q 1+$ pYES2.1-D308E PDSS1

$\triangle c o q 1+$ pYES2.1-WT COQ1

$\Delta$ coq1 + pYES2.1-D365E COQ1

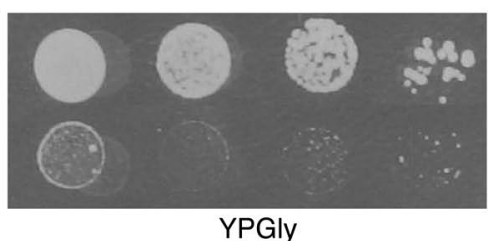

$\triangle$ coq2 + pYES2.1-WT-hCOQ2

$\triangle \operatorname{coq} 2+$ pYES2.1-m-hCOQ2

\section{Figure 6}

Functional complementation of yeast coq1- and coq2-null mutant. Growth of coq1- and coq2-null mutants on glucose (without amino acids, supplemented with histidine, leucine, and methionine, $20 \mathrm{~g} / \mathrm{l}$ glucose) or glycerol medium (YPGly, $20 \mathrm{~g} / \mathrm{l}$ glycerol) was compared. (A) The yeast $\Delta$ coq1-null mutant transformed with the wild-type and mutant (D308E) human PDSS1 cDNAs and the wild-type and mutant (D365E) yeast COQ1 genes on the pYES2.1 plasmid. (B) The yeast $\triangle$ coq2-null mutant transformed with the wild-type and mutant (m) human COQ2 genes on the pYES2.1 plasmid. The 4 spots for each experiment correspond to successive dilutions of transformed yeasts $(1,0.1,0.01$, and 0.001$)$. 
Table 3

Primers used for PCR amplification, sequencing, and cloning

$\begin{array}{lc}\text { Name of primer } & \text { Primer sequence } \\ \text { Human PDSs1 intronic primers } \\ 1 \mathrm{~F} & \text { GCAAGCGAGGAAGAGCGAAC } \\ 1 \mathrm{R} & \text { TCCCATTCATTCCGCCCACTG } \\ 2 \mathrm{~F} & \text { TGAGGCCTGTAAGCCCTTC } \\ 2 \mathrm{R} & \text { CCCCTTGCACCTCCCAAATC } \\ 3 \mathrm{~F} & \text { GCAATCTACCTGTAAATGGG } \\ 3 \mathrm{R} & \text { CTCCCCATCGCTACAAAAAA } \\ 4 \mathrm{~F} & \text { GGCTGAATTTCCGTATCTG } \\ 4 \mathrm{R} & \text { AACAGAGTGAGACTCCAGCT } \\ 5 \mathrm{~F} & \text { CAACAAGAGCGAACTCCGT } \\ 5 \mathrm{R} & \text { CCCAGCCGGTATATGCTAAT } \\ 6 \mathrm{~F} & \text { CCGATGTCCAGTTTCACAA } \\ 6 \mathrm{R} & \text { TCTGAGAAACAATGCGGTC } \\ 7-8 \mathrm{~F} & \text { CCTTCCAGTCAGTTCATCA } \\ 7-8 \mathrm{R} & \text { CTCTTGGGATTTTGGGTAC } \\ 9-10 \mathrm{~F} & \text { AAGGAATTCCTTCAGCCAGG } \\ 9-10 \mathrm{R} & \text { GCAATCTTCCCATCAGCTGT } \\ 11 \mathrm{~F} & \text { CCCACATCATCTAGACACTT } \\ 11 \mathrm{R} & \text { GTCACATCAAGAGTTCACCA } \\ 12 \mathrm{~F} & \text { GCAGGAAGTAAAACGCTGA } \\ 12 \mathrm{R} & \text { GACTTCAATGCAGACTTCAC }\end{array}$

PDSS1 cDNA primers

$\begin{array}{ll}\text { PDSS1-F } & \text { ATGGCCTCGCGCTGGTGG } \\ \text { PDSS1-R } & \text { TCATTTATCTCTTGTGAG }\end{array}$

TTATCTTGTGAG

\section{Yeast C0Q1 primers}

$\begin{array}{ll}\text { COQ1-mutF } & \text { TTCCAACTCGTAGATGA(G)ATGCTTGATTTTACTGT } \\ \text { COQ1-mutR } & \text { ACAGTAAAATCAAGCAT(C)TCATCTACGAGTTGGAA } \\ \text { COQ1-1 } & \text { ATGTTTCAAAGGTCTGGCGCT } \\ \text { COQ1-1419 } & \text { CTTTCTTCTTCTTAGTATACT }\end{array}$

\section{Human COQ2 intronic primers}

$\begin{array}{cl}1 \mathrm{~F} & \text { CGGGTHCCACTGCGCATGCCT } \\ 1 \mathrm{R} & \text { GGAGCCGACTCGGAGGCTGCT } \\ 2 \mathrm{~F} & \text { TAACTGACTTAAATTTGTACC } \\ 2 \mathrm{R} & \text { CACTATGTTATTCTGCAGAAT } \\ 3 \mathrm{~F} & \text { TGTTTAAATAAATGATTGAGTCA } \\ 3 \mathrm{R} & \text { CCTATTTTCTCCATTTCAAAG } \\ 4 \mathrm{~F} & \text { ATTTCGTGGTTTCTAAGAGGT } \\ 4 \mathrm{R} & \text { CTCCATAAAAGTGTAGTTTGC } \\ 5 \mathrm{~F} & \text { CCCTTTAGGATTCTAAAGTA } \\ 5 \mathrm{R} & \text { ATTTGGTTCTTAAAAACAGCA } \\ 6 \mathrm{~F} & \text { GAATTTTATGGTGTGTGACT } \\ 6 \mathrm{R} & \text { TTATACCAAGGAATATGAGG } \\ 7 \mathrm{~F} & \text { TTTGTTATGACCCAAGTCCA } \\ 7 \mathrm{R} & \text { GTATCAGATTTTGTATTCAAATC }\end{array}$

\section{COQ2 CDNA primers}

$\begin{array}{ll}\text { COQ2-1 } & \text { ATGACCCCAATTTCACAAGTA } \\ \text { COQ2-1114 } & \text { ATTTTCTATTTTATTCTCTA } \\ \text { COQ1-mutF } & \text { TTTAGGGATTGTCC()TGGGATTTGTGGAAA } \\ \text { COQ1-mutR } & \text { TTTCCACAAATTCCCA()GGACAATCCCTAAA }\end{array}$

Empty parentheses indicate the position of deleted nucleotide.

eral neuropathy, and mental retardation similar to that of her brother. She also has livedo reticularis and mildly elevated plasma lactate levels (3.2 nmol/1; N, 1.0-1.55) and lactate/pyruvate ratios (21; N, 6-14).

Patient 3, a girl, was born to healthy parents of French origin. Both parents were from the same geographical region and shared the same pat- ronymic, but they were not aware of any consanguinity. She presented neurologic distress at birth. Liver failure and nephrotic syndrome were diagnosed at 2 days of age. At 6 days, she developed anemia, pancytopenia, insulin-dependent diabetes, cytolysis, and seizures and presented hyperlactatemia $(22.7 \mathrm{mmol} / \mathrm{l})$ and high lactate/pyruvate molar ratios. She died at 12 days of age from multiorgan failure. Her older brother experienced collapse with hyperglycemia $(15 \mathrm{mmol} / \mathrm{l})$ and hyperlactatemia shortly after birth $(16 \mathrm{mmol} / \mathrm{l})$. He also had severe anemia, liver failure, and renal insufficiency and died at the age of 1 day.

Enzyme studies. Polarographic tests and/or spectrophotometric OXPHOS enzyme assays were carried out on skeletal muscle mitochondria, muscle homogenate, liver homogenate, cultured skin fibroblasts, or circulating lymphocytes, as previously described (25).

Genotyping. Fluorescent microsatellite markers of the Généthon database were used (26). The purified dye-labeled fragments were separated according to size on Amersham Biosciences MegaBACE 1000 96-capillary sequencers.

Analysis of $C o Q_{10}$ content and biosynthesis in skin fibroblasts. All solvents (Merck) were HPLC grade. Quinones were extracted from freeze-thawed fibroblasts $\left(10^{6}\right.$ cells in $1 \mathrm{ml}$ of water) according to the method of Folch et al. (27), with $3 \mathrm{ml}$ of chloroform/methanol (2:1) and 5 pmol of $\mathrm{CoQ}_{6}$ (Sigma-Aldrich) as internal standard. After shaking and centrifugation (2,000 g; 5 minutes), the organic layer was evaporated under a nitrogen stream at $60^{\circ} \mathrm{C}$ and resuspended in $100 \mu \mathrm{l}$ of methanol. CoQ ${ }_{10}$ was quantified by liquid chromatography/atmospheric pressure chemical ionization tandem mass spectrometry using a triple quadruple tandem mass spectrometer (API3000; Applied Biosystems) coupled with a liquid chromatographic system (HP 1100 series; Agilent Technologies). Lipid extracts $(20 \mu \mathrm{l})$ were injected into an Uptisphere ODB column $(33 \times 2 \mathrm{~mm}$, $3 \mu \mathrm{m}$; Interchim) and separated by a linear gradient from $40 \%$ solvent $\mathrm{A}$ (methanol/water, 9:1) to $90 \%$ solvent B (chloroform/methanol, 1:1) at $500 \mu \mathrm{l} / \mathrm{min}$ for 2 minutes for a total run time of 5 minutes (column oven maintained at $35^{\circ} \mathrm{C}$ ). Multiple-reaction monitoring transitions were 863.9-197.2 and 591.5-197.2 for $\mathrm{CoQ}_{10}$ and $\mathrm{CoQ}_{6}$, respectively. CoQ 10 content was expressed with reference to the protein content of the cell extracts. Cells were labeled with $\left[{ }^{3} \mathrm{H}\right]$ mevalonate, and extraction of lipids and HPLC analyses were performed as previously described (6). Since decaprenyl-PP cannot be separated by reverse-phase HPLC, both phases of lipid extracts (petroleum ether and methanol) were combined, dried, and subjected to treatment with wheat germ acid phosphatase (28). Decaprenol was identified on the HPLC chromatogram by a reference sample and mass spectrometry.

Mutation screening. The exons and exon-intron boundaries of the human prenyldiphosphate synthase (PDSS1) and OH-benzoate polyprenyltransferase (COQ2) genes were PCR amplified using specific intronic primers (Table 3). Amplification products were directly sequenced using the PRISM Ready Reaction Sequencing Kit (Applied Biosystems) on an automatic sequencer (Applied Biosystems).

Complementation of a yeast coq1-and coq2-null mutant. The COQ1 gene was PCR amplified using oligonucleotide primers COQ1-1 and COQ1-1419 and cloned in a PYES2.1 plasmid according to the manufacturer's recommendations (Invitrogen). The D365E mutation was introduced into the wild-type yeast COQ1 gene cloned in PYES2.1 using the QuikChange XL site-directed mutagenesis kit (Stratagene) and COQ1-mutF and COQ1-mutR primers (Table 3; the mutation is shown in parentheses). Human wild-type and mutant PDSS1 cDNAs were RT-PCR amplified from control and patient fibroblast RNA using PDSS1-F and PDSS1-R primers (Table 3) and cloned in a pYES2.1 plasmid. Human wild-type COQ2 cDNA previously cloned in a pBV134.nuc vector (gift from I. Climent, Biovitrum, Stockholm, Sweden) was PCR amplified using COQ2-1 and COQ2-1114 primers and cloned in PYES2.1 plasmid. The c.1198delT, N401fsX415 mutation was introduced into the wild-type COQ2 
cDNA using the site-specific mutagenesis kit (Stratagene) and COQ2-mutF and COQ2-mutR primers (Table 3; the deletion is shown in parentheses).

The yeast $\Delta \operatorname{coq} 1$ and $\Delta \operatorname{coq} 2$ strains (his ${ }^{-}$, ura $^{-}$, leu-', met-) were transformed according to ref. 29 , and the resulting clones were grown on yeast base plates without amino acids supplemented with histidine, leucine, and methioine and replicated on a glycerol-rich medium for 3-4 days at $30^{\circ} \mathrm{C}$.

\section{Acknowledgments}

We thank Isabel Climent (Biovitrum) for providing us with human COQ2 cDNA. This research was supported in part by the
Association Française contre les Myopathies and by the Fondation Jérôme Lejeune.

Received for publication May 15, 2006, and accepted in revised form December 5, 2006.

Address correspondence to: Agnès Rötig, INSERM U781 and Department of Genetics, Hôpital Necker-Enfants Malades, 149 rue de Sèvres, 75105 Paris, France. Phone: 33-144-49-51-61; Fax: 33-147-34-85-14; E-mail: roetig@necker.fr.
1. Turunen, M., Olsson, J., and Dallner, G. 2004. Metabolism and function of coenzyme Q. Biochim. Biophys. Acta. 1660:171-199.

2. Ogasahara, S., Engel, A.G., Frens, D., and Mack, D. 1989. Muscle coenzyme Q deficiency in familial mitochondrial encephalomyopathy. Proc. Natl. Acad. Sci. U. S. A. 86:2379-2382.

3. Sobreira, C., et al. 1997. Mitochondrial encephalomyopathy with coenzyme Q10 deficiency. Neurology. 48:1238-1243.

4. Boitier, E., et al. 1998. A case of mitochondrial encephalomyopathy associated with a muscle coenzyme Q10 deficiency. J. Neurol. Sci. 156:41-46.

5. Di Giovanni, S., et al. 2001. Coenzyme Q10 reverses pathological phenotype and reduces apoptosis in familial CoQ10 deficiency. Neurology. 57:515-518.

6. Rotig, A., et al. 2000. Quinone-responsive multiple respiratory-chain dysfunction due to widespread coenzyme Q10 deficiency. Lancet. 356:391-395.

7. Rahman, S., Hargreaves, I., Clayton, P., and Heales, S. 2001. Neonatal presentation of coenzyme Q10 deficiency. J. Pediatr. 139:456-458.

8. Van Maldergem, L., et al. 2002. Coenzyme Qresponsive Leigh's encephalopathy in two sisters. Ann. Neurol. 52:750-754.

9. Musumeci, O., et al. 2001. Familial cerebellar ataxia with muscle coenzyme Q10 deficiency. Neurology. 56:849-855.

10. Lamperti, C., et al. 2003. Cerebellar ataxia and coenzyme Q10 deficiency. Neurology. 60:1206-1208.

11. Quinzii, C., et al. 2006. A mutation in parahydroxybenzoate-polyprenyl transferase (COQ2) causes primary coenzyme Q10 deficiency. Am.J. Hum. Genet. 78:345-349.

12. Lopez, L.C., et al. 2006. Leigh syndrome with nephropathy and CoQ10 deficiency due to decaprenyl diphosphate synthase subunit 2 (PDSS2) mutations. Am. J. Hum. Genet. 79:1125-1129.

13. Ashby, M.N., and Edwards, P.A. 1990. Elucidation of the deficiency in two yeast coenzyme Q mutants. Characterization of the structural gene encoding hexaprenyl pyrophosphate synthetase. J. Biol. Chem. 265:13157-13164.

14. Fujisaki, S., Hara, H., Nishimura, Y., Horiuchi, K., and Nishino, T. 1990. Cloning and nucleotide sequence of the ispA gene responsible for farnesyl diphosphate synthase activity in Escherichia coli. J. Biochem. (Tokyo). 108:995-1000.

15. Math, S.K., Hearst, J.E., and Poulter, C.D. 1992 The crtE gene in Erwinia herbicola encodes geranylgeranyl diphosphate synthase. Proc. Natl. Acad. Sci. U. S. A. 89:6761-6764

16. Cunillera, N., et al. 1996. Arabidopsis thaliana contains two differentially expressed farnesyl-diphosphate synthase genes. J. Biol. Chem. 271:7774-7780.

17. Carattoli, A., Romano, N., Ballario, P., Morelli, G., and Macino, G. 1991. The Neurospora crassa carotenoid biosynthetic gene (albino 3 ) reveals highly conserved regions among prenyltransferases. J. Biol. Chem. 266:5854-5859.

18. Liang, P.H., Ko, T.P., and Wang, A.H. 2002. Structure, mechanism and function of prenyltransferases. Eur. J. Biochem. 269:3339-3354.

19. Song, L., and Poulter, C.D. 1994. Yeast farnesyldiphosphate synthase: site-directed mutagenesis of residues in highly conserved prenyltransferase domains I and II. Proc. Natl. Acad. Sci. U. S. A. 91:3044-3048.

20. Koyama, T., et al. 1996. Identification of significant residues in the substrate binding site of Bacillus stearothermophilus farnesyl diphosphate synthase. Biochemistry. 35:9533-9538.

21. Joly, A., and Edwards, P.A. 1993. Effect of sitedirected mutagenesis of conserved aspartate and arginine residues upon farnesyl diphosphate synthase activity. J. Biol. Chem. 268:26983-26989.

22. Forsgren, M., et al. 2004. Isolation and functional expression of human COQ2, a gene encoding a polyprenyl transferase involved in the synthesis of CoQ. Biochem. J. 382:519-526.

23. Gin, P., and Clarke, C.F. 2005. Genetic evidence for a multi-subunit complex in coenzyme $\mathrm{Q}$ biosynthesis in yeast and the role of the Coq1 hexaprenyl diphosphate synthase. J. Biol. Chem. 280:2676-2681.

24. Marbois, B., et al. 2005. Coq3 and Coq4 define a polypeptide complex in yeast mitochondria for the biosynthesis of coenzyme Q. J. Biol. Chem. 280:20231-20238.

25. Rustin, P., et al. 1994. Biochemical and molecular investigations in respiratory chain deficiencies. Clin. Chim. Acta. 228:35-51.

26. Dib, C., et al. 1996. A comprehensive genetic map of the human genome based on 5,264 microsatellites. Nature. 380:152-154.

27. Folch, J., Lees, M., and Sloane Stanley, G.H. 1957. A simple method for the isolation and purification of total lipides from animal tissues. J. Biol. Chem. 226:497-509.

28. Carson, D.D., and Lennarz, W.J. 1981. Relationship of dolichol synthesis to glycoprotein synthesis during embryonic development. J. Biol. Chem. 256:4679-4686.

29. Gietz, D., St. Jean, A., Woods, R.A., and Schiestl, R.H. 1992. Improved method for high efficiency transformation of intact yeast cells. Nucleic Acids Res. 20:1425. 